\title{
Mice learn to avoid the rat race
}

\section{Mice can learn to overcome their naturally aggressive approach to conflict resolution, instead adopting a cooperative strategy. This discovery provides a simple animal model in which to investigate a complex social behaviour.}

\section{SCOTT M. RENNIE \& MICHAEL L. PLATT}

$\mathrm{S}$ ocial interactions are often complicated by conflicts of interest. Humans and other animals adopt diverse strategies to resolve such disputes. Stronger individuals can often secure their interests at the expense of weaker individuals, but this strategy can be costly if it requires aggression. Strategies that are more cooperative and egalitarian can also develop among kin $^{1}$ or individuals who reciprocate in repeated interactions ${ }^{2}$. Theoretical and experimental studies suggest that cooperation depends on cognitive control processes that override the impulse to acquire tangible rewards ${ }^{3}$. This theory now finds support from Choe et al. ${ }^{4}$, writing in Nature Communications. The authors demonstrate that pairs of mice can learn to coordinate their behaviour to achieve an egalitarian distribution of rewards - but only when rewards are delivered directly to the brain, rather than through food.

Mice are flexible in their social behaviour. At low population densities, they establish and aggressively defend territories, whereas at higher population densities, they develop strict hierarchies in which a single male dominates several subordinates ${ }^{5}$. Neither of these strategies whiffs of cooperation. For male mice, as for many other animals, size, aggressiveness and persistence strongly determine social rank. Mice decide whether to compete by comparing potential costs and benefits on the basis of perceived asymmetries in these qualities. These computations rely on a neural circuit that connects two brain regions - the mediodorsal thalamus and the dorsomedial prefrontal cortex ${ }^{6}$.

Choe et al. set out to investigate whether mice have the capacity to override their natural tendencies towards dominance-based conflict resolution. To do this, they developed a clever coordination task. They trained mice to enter a central start zone in a three-chambered box, and then to follow a visual cue to either the left or right chamber of the box to receive a reward. Next, they paired trained animals to take the trial together. When both mice occupied the start zone, a trial was initiated (Fig. 1a). The first mouse to enter the correct chamber received a reward of either food pellets or wireless brain stimulation (WBS)

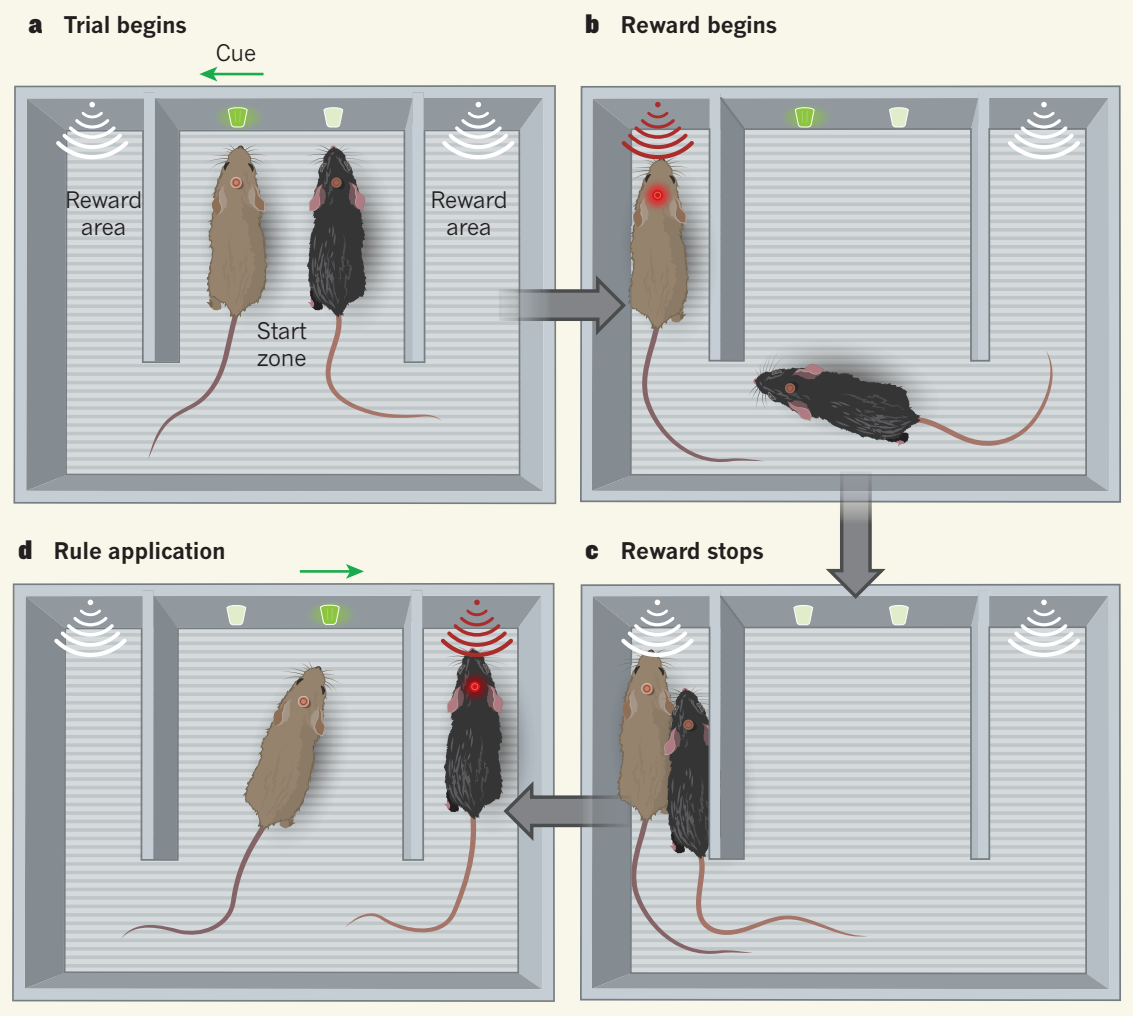

Figure 1 | Mice learn a rule to resolve conflict. a, Choe et al. ${ }^{4}$ taught mice that entering a start zone in a three-chambered box would trigger a trial, in which a visual (light) cue would indicate where to go to receive a reward - into either the left- or right-hand reward area. Once trained, mice performed the task in pairs. Both animals had to occupy the start zone to initiate a trial. $\mathbf{b}$, The first animal to enter the cued reward zone received a reward of wireless brain stimulation of the medial forebrain bundle (red circle). c, If the second mouse entered the reward zone, the reward was terminated. $\mathbf{d}$, The authors showed that the pairs learnt to observe a side-allocation rule, in which one followed the cue to rewards in the left-hand chamber, the other to those in the right. This increased overall reward and equity between the animals.

of the medial forebrain bundle - a region that, when stimulated, can override all other rewards, including food, water and $\operatorname{sex}^{7}$ (Fig. 1b). In the WBS trials, the reward was terminated if the second mouse entered the chamber (Fig. 1c), although this was not possible in the food trial.

As expected, when mice were rewarded with food pellets, dominant ones coerced their subordinate partners into the start zone to enable the trial to begin, and then monopolized the rewards. By contrast, Choe and colleagues found that most animals that were rewarded with WBS developed and maintained a simple alternate-side-allocation rule: each mouse in a pair monopolized only one reward chamber and avoided the other (Fig. 1d). As a result, one mouse gained rewards in trials when the left-hand chamber was the reward chamber, and the other gained rewards when the righthand chamber was the reward chamber. By following this rule, mice increased both the total amount of reward received and the equality with which that reward was divided.

Remarkably, WBS seemed to override the hierarchical, despotic behaviour that 
developed over food rewards. Rule-following mice in WBS trials displayed very little aggression, and the limited aggression observed had minimal impact on choice behaviour. Asymmetries in the sizes of the paired animals, which are a key determinant of social status, also had no effect on WBS-induced cooperation. Even when the authors reshuffled the mice into new pairs in which both animals had the same side preference (both monopolizing the left chamber in their previous trials, for instance), the animals rapidly re-established the alternate-side-allocation rule - thus demonstrating remarkable flexibility.

Choe and colleagues' experiments indicate that certain factors can put natural limitations on cooperation. These include food depriva$\operatorname{tion}^{8}$ and the presence of a powerful appetitive stimulus, the food pellet, which was clearly visible in the food-reward trials, and was presumably aromatic, too. By contrast, although WBS was associated with a light cue, it was otherwise not obvious to the unrewarded animal. These findings resonate with previous studies showing that the physical presence of tangible rewards impairs delayed gratification in blue jays ${ }^{9}$, complex rule-following by monkeys ${ }^{10}$ and chimps ${ }^{11}$, and cooperation in humans ${ }^{12}$.

The current study raises several questions. First, is social coordination by rule-following supported by the same neural circuit between the mediodorsal thalamus and dorsomedial prefrontal cortex that underlies status-based conflict resolution? If not, perhaps WBS overrides this circuit by triggering different circuits that stamp in a more 'cognitive' strategy.

Second, what role do internal states, such as hunger, have in strategy selection? The mice in Choe and colleagues' WBS trials were not food-deprived, and it would be interesting to determine how hunger would affect their behaviour.

And third, to what extent is rule-based coordination social at all? Determining to what extent this coordination depends on physical similarity between partners, transmission of social signals, or the implementation of a similar computational routine could provide clues to this question. If WBS could elicit the same type of coordination between a mouse and a robot, for example, this would demonstrate that the behaviour observed in the current study does not involve any sort of attribution of agency or strategic thinking, and instead arises from pure associative learning. Could WBS drive cooperation between the cartoon characters Tom the cat and Jerry the mouse, or might it just stop them from fighting?

Choe et al. have provided a compelling demonstration of a transition from aggressive to more-egalitarian interactions, at a time when examples of cooperation between animals in the laboratory are controversial and rare $^{8}$. Crucially, they have done so in mice, an animal model that will allow the whole range of powerful techniques in the neuroscience toolbox, from behaviour tracking to molecular-genetic tools such as optogenetics to electrophysiology, to be brought to bear on the these tricky but important social questions.

Scott M. Rennie is in the Champalimaud Neuroscience Programme, Champalimaud Centre for the Unknown, 1400-038 Lisbon, Portugal. Michael. L. Platt is in the Departments of Neuroscience, of Psychology and of Marketing, University of Pennsylvania, Philadelphia, Pennsylvania 19104, USA. e-mails:scott.rennie@neuro.fchampalimaud.org; mplatt@pennmedicine.upenn.edu
1. Hamilton, W. D. Am. Nat. 97, 354-356 (1963).

2. Trivers, R. L. O. Rev. Biol. 46, 35-57 (1971).

3. Stevens, J. R. \& Hauser, M. D. Trends Cogn. Sci. 8, 60-65 (2004)

4. Choe, I.-H. et al. Nature Commun. 8, 1176 (2017)

5. Wang, F., Kessels, H. W. \& Hu, H. Trends Neurosci. 37, 674-682 (2014)

6. Zhou, T. et al. Science 357, 162-168 (2017).

7. Olds, J. \& Milner, P. J. Comp. Physiol. Psychol. 47, 419-427 (1954).

8. Viana, D. S., Gordo, I., Sucena, E. \& Moita, M. A. P. PLOS ONE 5, e8483 (2010).

9. Stephens, D. W., McLinn, C. M. \& Stevens, J. R Science 298, 2216-2218 (2002).

10.Silberberg, A. \& Fujita, K. J. Exp. Anal. Behav. 66, 143-147 (1996).

11.Boysen, S. T. \& Berntson, G. G. J. Exp. Psychol. Anim. Behav. Processes 21, 82-86 (1995).

12.Nishi, A., Shirado, H., Rand, D. G. \& Christakis, N. A. Nature 526, 426-429 (2015).

This article was published online on 21 December 2017.

\section{MICROBIOLOGY}

\section{Pathogens boosted by food additive}

Epidemic strains of the bacterium Clostridium difficile have now been found to grow on unusually low levels of the food additive trehalose, providing a possible explanation for C. difficile outbreaks since 2001. SEE ARTICLE P.291

\section{JIMMY D. BALLARD}

B etween 2001 and 2006, epidemic strains of the bacterium Clostridium difficile, which can inhabit the bowel and cause dangerous diarrhoea, unexpectedly emerged in the United States, Canada and several European countries ${ }^{1,2}$. Most of these strains originated from a single lineage of $C$. difficile known as ribotype 027 (RT027; ref. 2), which has now spread around the world ${ }^{3}$. Of particular concern has been the correlation between RT027 and a dramatic increase in deaths related to $C$. difficile $e^{4}$. The mystery of why this ribotype and a second one, RT078, became so prevalent apparently out of thin air has remained largely unsolved ${ }^{5}$. On page 291, Collins et al. ${ }^{6}$ raise the possibility that the seemingly harmless addition of a sugar called trehalose to the food supply contributed to this disease epidemic.

Collins and colleagues first explored how RT027 and RT078 grow, by comparing carbon-source preferences between strains of C. difficile. They noted a peculiar property of these two lineages - they can use low concentrations of trehalose as a sole source of carbon. Next, the authors analysed the genomes of RT027 and RT078, and discovered that each encodes unusual sequences that might explain their ability to grow in low levels of trehalose.

The researchers showed that RT027 carries a single-nucleotide genetic variant that changes an amino-acid residue in the protein TreR from leucine to isoleucine. Tre $\mathrm{R}$ is a transcriptional repressor that is inhibited by trehalose. When active, TreR prevents expression of the gene tre $A$, which encodes a phosphotrehalase enzyme involved in metabolizing trehalose into glucose and glucose derivatives. Thus, trehalose is metabolized only when its levels are high enough to inhibit TreR. Collins et al. propose that the mutation in RT027 changes TreR's affinity for trehalose and allows it to be repressed by substantially lower levels of the sugar than normal. This frees the TreA protein to metabolize trehalose and allows RT027 to grow on low levels of the sugar (Fig. 1).

By contrast, RT078 has adapted to grow on low amounts of trehalose by acquiring four genes involved in trehalose uptake and metabolism. The genes encode second copies of TreR and TreA, a trehalose transporter protein dubbed PtsT that helps cells take up the sugar, and another enzyme, TreX, involved in trehalose metabolism. Unexpectedly, RT078 does not share the genetic alteration in TreR that is found in RT027. As Collins and colleagues point out, it therefore seems that two epidemic strains of C. difficile have optimized trehalose metabolism in unrelated ways.

The investigators next provided evidence that trehalose metabolism directly relates to enhanced virulence of RT027 in vivo. First, they showed that deleting treA in RT027 and thereby preventing trehalose metabolism 